\title{
Paraganglioma of the ponytail in one case
}

\begin{abstract}
Paraganglioma is a rare neuroendocrine tumor composed of paraganglionar cells, much vascularized, usually benign. It is located mainly in the medullo-adrenal part of the superior gland, the carotid glomus and the jugular glomus. Paraganglioma of the ponytail is very rare. The first case was reported in 1972, the clinical signs are not specific. The magnetic resonance image (MRI) is the choice test. It appears in isosignal in sp T1 and in isosignal in T2, containing several sinuous vessels which represent a diagnostic value of this tumor. This case of the ponytail paraganglioma has been confirmed by MRI and immunohistochemistry.
\end{abstract}

Keywords: paraganglioma, ponytail, MRI, immunohistochemistry
Volume I Issue 3 - 2017

\author{
Belhachmi A, Gazzaz M, El Mostarchid B \\ Department of Neurosurgery, MohamedV Military Hospital, \\ Morocco
}

Correspondence: Belhachmi A, Department of Neurosurgery, MohamedV Military Hospital, Hay Ryad, Rabat, Morocco, Fax (2I2) 0376834 40,Email adil.belhachmi@hotmail.com

Received: August 24, 2017 | Published: November 16, 2017

\section{Introduction}

Paraganglioma is a rare neuroendocrine tumor, composed of paraganglionic cells, much vascularized, usually benign. It is located mainly in the adrenal medulla, carotid glomus and jugular glomus. Paraganglioma of the ponytail is very rare. The first case of the ponytail was reported in 1972.

\section{Observation}

A 19-year-old patient with no significant medical or surgical pathological antecedents who presented 5-month-old chronic, intermittent low back pain that became more and more intense, aggravated by lumbar lungosciatalgia L5, with heaviness of the lower left limb and walking, without sphincteric disorders, nor saddle anesthesia, nor trophic disorders. Clinical examination showed a distal paresis of the foot ghe, the patient does not hold the maingazini, hypotone member. The osteo-tendinous reflexes were abolished, babinski positive, on the other hand the somatic examination was without particularities. The standard radiography of the lumbar spine was normal. $^{1-3}$

Lumbar magnetic resonance (MRI) images revealed an intravertebral lesion in relation to oval L2 measuring $28 / 18 \mathrm{~mm}$. This lesion was iso signaling in $\mathrm{T} 1$ and isosignal in $\mathrm{T} 2$ with sepentigine vessels (Figure $1 \& 2$ ).

An Iaminectomy of L3-L2 was performed. After opening of the dura mater, we discover a tumor of 2.8 by 1.8 centimeter, fleshy red, intimately adherent to sinuous vessels. The excision of the tumor was monobloc. With sacrifice of the nerve root carrier L5. The postoperative follow-up was uncomplicated, with immediate disappearance of pain and progressive recovery of muscle strength and walking. ${ }^{4-6}$

The histological study of the resection showed: A fragment weighing $5 \mathrm{gms}$ and measuring $3 \times 2 \times 1.5 \mathrm{~cm}$; to the cut appearance white-grayish well limited by a capsule with some haemorrhagic rearrangements, the capsule is very fine. The microscopic study deals with a tumor proliferation well limited by a fibrous capsule, endocrine architecture, made of cells with clear cytoplasm sometimes eosinophilic to the regular rounded nucleus comprising a fine chromatin. In rare places; A few multinucleated cells are observed. There are no observed mitotic figures; the stroma is fibrovascular, in large foci becoming fibrohyalin (Figure $3 \& 4$ ).

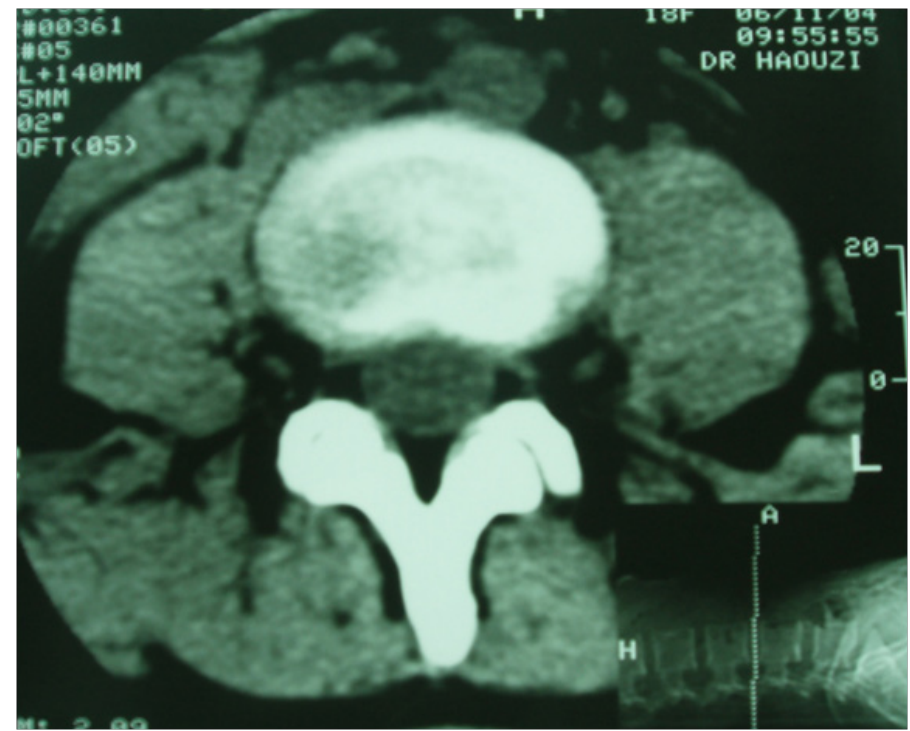

Figure I Image of CT in axial section C- showing a well-limited hypodence tumor, filling the dural fossa.

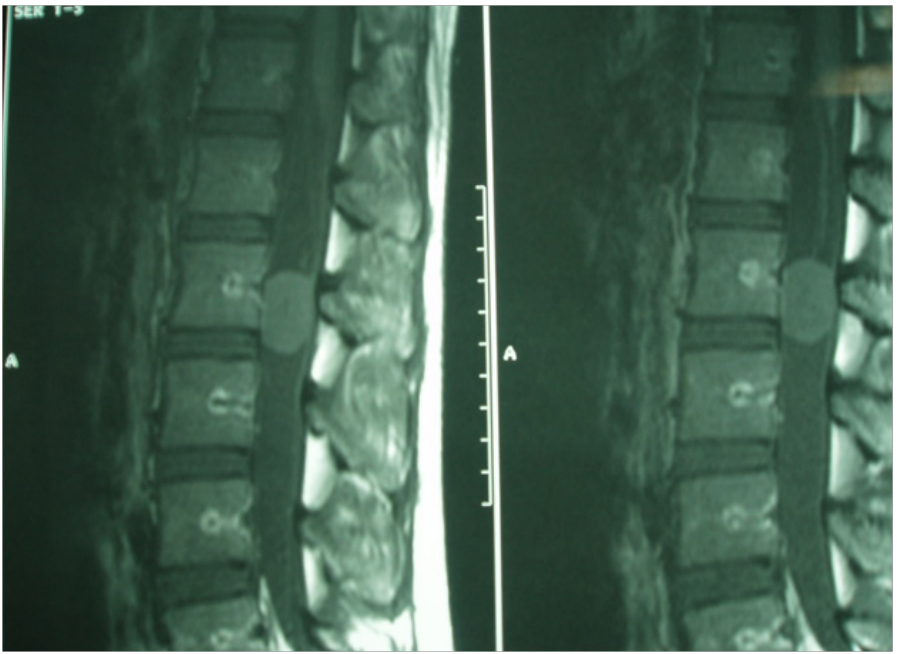

Figure 2 Sagittal MRI scan in sp TI showing the intradural lesion, in issosignale at the L2 level. 


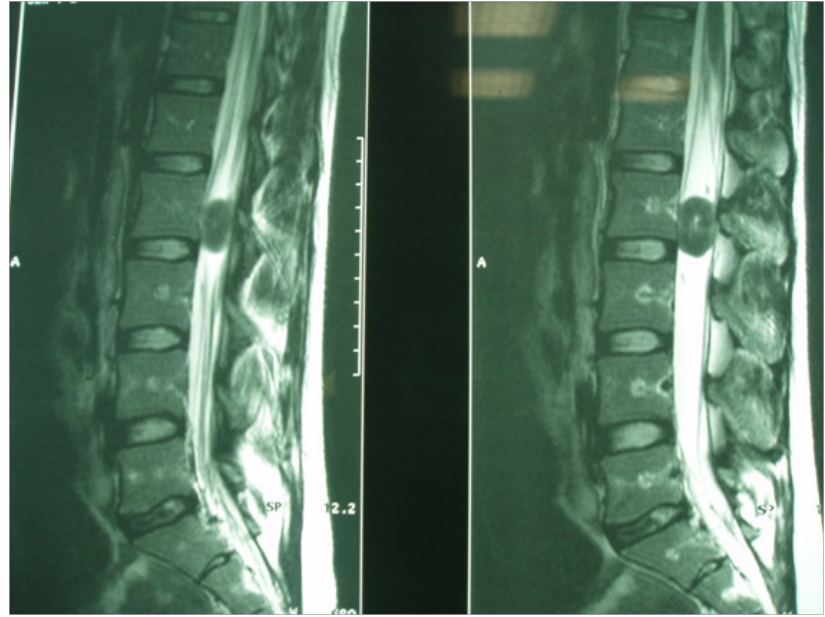

Figure $3 \mathrm{MRI}$ image in sagittal section in T2 showing intradural lesion, isosignal at L2 level.

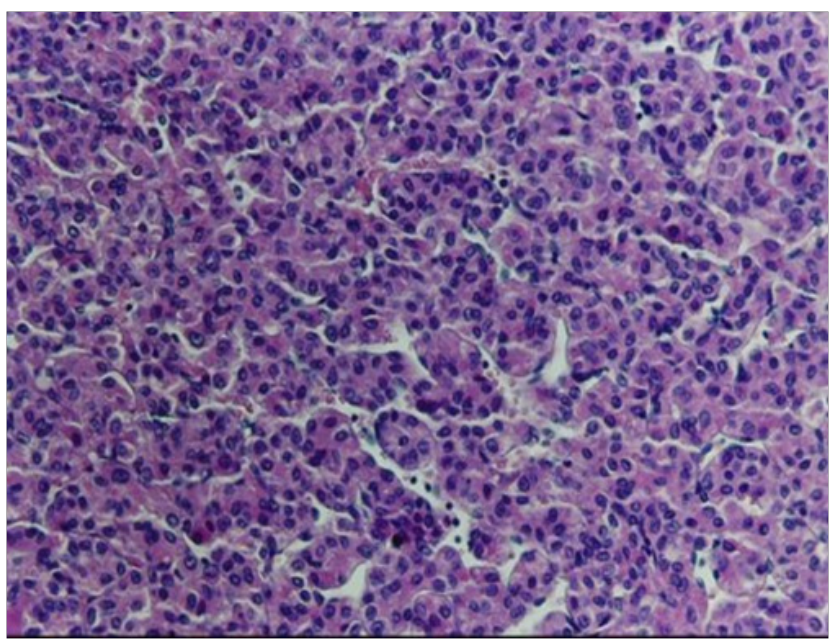

Figure 4 Anatomopathological microscopic image showing grouped monomorphic cells, partially surrounded by the vascular septum, the "zellaballen model" (Hematoxylin and Eosin X200).

\section{An immunohistochemical study has been} carried out which has objectified
a) Chromogranin (+)
b) Synaptophysime (+)
c) NSE (+)
d) $\mathrm{CD}(-)$

\section{Discussion}

Paraganglioma is also known as parenchymal paraganglioma or pheochromocytoma. Paraganglioma of the ponytail is very rare. Paraganglioma ponytail is usually seen, in the fourth dicenny or beyond, with predominantly male. It manifests itself in low back pain and then lumbosciatalgia with motor or sensory deficit of the leg, urinary or fecal incontinence (ponytail syndrome).

The symptom of cushing has been reported with other sites of the paraganglionary tumor, but exceptionally in the pony tail. Preoperative diagnosis of paraganglioma is very difficult. MRI is the exam of choice. It is in iso or hyper signal in T1 and the isosignal in T2 with sinuous vessels have been highlighted as diagnostic value of this tumor. Having as a differential diagnoses radiologically schwannoma and ependymoma. ${ }^{7,8}$

Macroscopically, in peroperative, it is a fleshy red tumor, containing sinuous vessels. Clinical history data, patient age, lumbar spine, and macroscopic appearance are the main factors in the positive diagnosis of the ponytail paraganglioma. There are also other tumors within the framework of the differential diagnosis are: myxopapilloma of the ponytail. ${ }^{9}$

But immunohistochemistry has solved the problem of paragnaglioma, becoming positive to synaptophysime, and to chromogranin which are specific to paraganglioma. The immunochemical reaction for NSE is generally diffuse and strongly positive for the paraganglion cells, and negative for the ependymoma.

The search for the neurosecretory azyrophilic granules of paraganglioma, demonstrated by the Grimelius method, is more reliable, cheaper, and easily eliminates the ependymoma. For frequent ponytail tumors such as the pituitary astrocytoma is eliminated in front of the positive GFPA, and measuring the density of the vesicular nucleus under the electron microscope.

The ponytail paraganglioma is a benign tumor that evolves slowly. It is manifested by a progressive suffering of the roots of the ponytail; the treatment is mainly surgical by the complete resection making the prognosis satisfactory. But among the few complications a small percentage of resection of the filium terminal. Rare cases of intracranial metastasis and vertebral metastases. No studies of radiotherapy to prevent tumor recurrence have been demonstrated.

\section{Conclusion}

Abdominal and surrenal ultrasound without special features. Urine catecholamine's not made. After 6 months of evolution the patient was asymptomatic.

\section{Acknowledgements}

None.

\section{Conflict of interest}

Author declares no conflict of interest.

\section{References}

1. Aghakhani N, George BF, Parker. Paraganglioma of the ponytail. Two cases and review of the literature. Acta Neurochir (Wien). 1999;141:8187.

2. Araki Y, Ishida T, Ootan M, et al. MRI of the Ponytail Paraganglioma. Neuroradiology. 1993;35:232-233.

3. Castel JP, Cuny E, Boulan P, et al. Paraganglioma of the ponytail clinical aspects and MRI. About a case. Neurosurgery. 1995;41(2):112-115.

4. Lerman RI, Kaplan ES, Deaman L. The intradural Paraganglioma of the terminal filum Case report. J Neurosurg. 1972;36:652-658.

5. Peter C Burger, Bernand W Scheithauer. Anatomopathological atlas of the paraganglioma (Third series). USA; Tumors of the central nervous system of the Institution of the Public Forces: 1994. p. 317-320.

6. PH Roche, Figarella-Branger D, Regis J, et al. Ponytail paraganglioma with intracranial and intraspinal metastases. Accta Neurochir (Wien). 1996;138(4):475-479. 
7. Rickert CHS. The Probst-Cousin, Schumacher HW and a Paragangliome of the ponytail. Pathologe (Germany). 1997;18:484- 487.

8. Raftopoulos C, Brucher JM. Hemodynamic activity associated with Paraganglioma of the cauda equina. J Neurosurg. 1995;82(4):704-705.
9. Lipper S, Decker RE. Paraganglioma of the cauda equina. A histologic, immunohistochemical, and ultrastructural study and review of the literature. Surgical Neurology. 1984;22(4):415-420. 